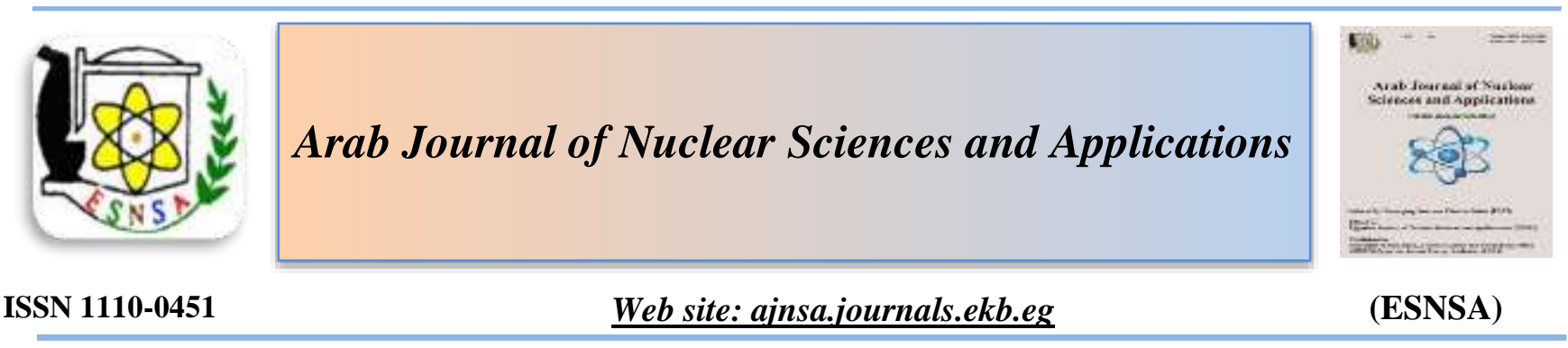

\title{
New Applications for Linear Induction Drives used for Silent Propulsion Systems in Nuclear Submarines and Supercarriers
}

\author{
Sherif M E Ismaeel ${ }^{1,2}$ \\ ${ }^{(1)}$ Physics Department, Faculty of Science, Ain Shams University, Cairo, Egypt \\ ${ }^{(2)}$ Physics Department, College of Sciences and Humanities, Prince Sattam Bin Abdulaziz University, Al- \\ Kharj, Saudi Arabia
}

Received 08 Aug 2019 Accepted 27 Aug 2019

\begin{abstract}
A 2-dimensional analytical and numerical theory for linear induction drives (LIDs) using hot seawater from coolant systems of nuclear reactors as a driving medium has been presented. This work suggests its use in nuclear submarines as it could be considered an important application on how to utilize the coolant output from nuclear reactors in nuclear submarines to generate enough force to drive nuclear submarines and supercarriers as silent propulsion systems. In this paper, the stepped distribution of the 3-phase winding of the LID has been presented by Fourier integrals. The boundary value problem of the LID has been solved using Fourier transform techniques. The force and seawater input power expressions of the LID have been given in Fourier integral forms, which permit studying the effect of the design parameters of the LID to get the optimum performance. The effect of changing the conductivity of seawater due to the change in seawater temperature has been studied, showing the necessity of injecting hot water from the coolant system of the nuclear submarines or supercarriers, into the LID to improve their performance. The paper shows that the duct size, the conductivity, and the frequency are crucial factors for controlling the silent speed of these machines. For the same length of the machine, the paper shows that decreasing the number of poles and increasing the pole pitch improves the performance than increasing the number of poles and decreasing the pole pitch.
\end{abstract}

Keywords: linear induction drives, linear induction motors, nuclear submarines.

\section{Introduction}

Nuclear propulsion research was first undertaken in the United States during the 1940s and has since yielded important engineering results, including the invention of the pressurized water reactor (PWR) and the development of significant safety practices [1]. Since the 1955 launch of the USS Nautilus, nuclear naval vessels have accrued over 12,000 reactor years of operational experience, demonstrating that with effective technology and training, nuclear marine propulsion can be a safe and reliable option [1]. Nuclear submarine propulsion system includes steam turbines, to which the steam is delivered from the cooling system of the reactor. These nuclear reactors give powers in the order of $550 \mathrm{MWt}$ (thermal MW). For example, the Nimitz class super-carriers using nuclear fission pressurized water reactors have two reactors rated at $550 \mathrm{MWt}$ each. Each reactor generates enough steam to produce a $100 \mathrm{MW}$ electricity supply plus 40,000 shaft horsepower [2]; they are expected to operate for about 20 years [3]. Now the primary mission of the naval submarine is to remain undetected and covert. Any vibrational radiation emanating from the submarine has the potential to give the submarine's presence, or even location, away, rendering the primary mission capability impossible [4]. This work, suggest the replacement

Corresponding author: sherifelhosainy77@hotmail.com

DOI: 10.21608/ajnsa.2020.15711.1250

(C) Scientific Information, Documentation and Publishing Office (SIDPO)-EAEA 
of steam turbines with a LID, which would eliminate any kind of vibrations that can appear from the turbines.

As for linear induction machines (LIM), many authors have made several attempts [5-10] to get the best performance out of these types of machines, but one of the most important was in 1984, which made a crucial condition $[11,12]$, for solving the problem of extra high speed in linear induction motors and space launcher, viz.;

$$
\sigma H z=\text { constant }
$$

which will be used in this paper to solve the problem of vibration in submarines. Figure (1) shows the proposed position of the LID.

In this paper, a two dimensional analytical and numerical electromagnetic theory of the linear induction motors (LIMs) [13 - 15] has been applied to develop the theory of linear induction drives (LIDs). The solution is given using Fourier techniques. The stepped nature of the machine winding is fully presented mathematically by Fourier integrals, and is incorporated into the solution of the boundary value problem of the machine. The force and the seawater input power expressions are derived in Fourier integral form, which gives the opportunity of studying the effect of the different parameters, given in table I, of the machine on its performance.

The analytical and numerical solutions given in this paper represent a proposal being displayed, for driving systems as silent propulsion systems for both nuclear submarines and supercarriers, using hot seawater from the coolant system of small nuclear reactors as a driving medium. The effect of varying the conductivity, and the frequency and the duct size have been studied. Figure 2 shows that, as seawater temperature increases, the conductivity increases showing that for every increase by $1^{\circ} \mathrm{C}$, the conductivity increases in the order of $2-4 \%,[16$, 17].

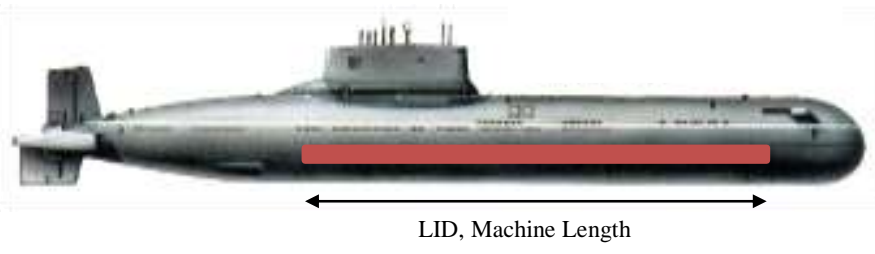

(a)

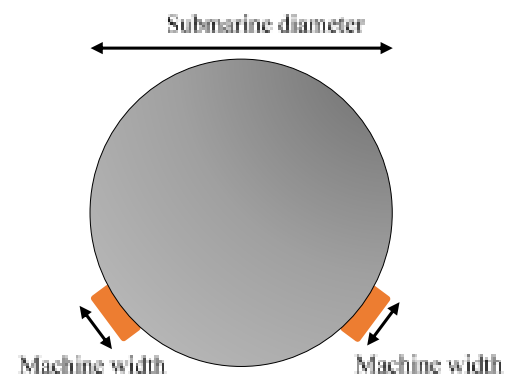

(b)

Fig. (1): The location of the LID on the nuclear submarine.

(a) Side view of the nuclear submarine showing the place of LID. (b) Cross-sectional view of nuclear submarine showing the LID

TABLE (I): LINEAR INDUCTION DRIVE PARAMETERS AND SYMBOLS

\begin{tabular}{rccc}
\hline Name & Symbol & $\begin{array}{c}\text { Long } \\
\text { Machine }\end{array}$ & $\begin{array}{c}\text { Short } \\
\text { Machine }\end{array}$ \\
\hline Frequency & $H z$ & $800 \mathrm{~Hz}$ & $800 \mathrm{~Hz}$ \\
number of phases & $m$ & 3 & 3 \\
$\begin{array}{r}\text { number of pole pairs } \\
\text { of the machine }\end{array}$ & $p$ & 32 & 4 \\
$\begin{array}{r}\text { number of slots per } \\
\text { pole per phase }\end{array}$ & $q$ & 16 & 8 \\
Pole pitch & $\tau$ & $1.92 \mathrm{~m}$ & $0.96 \mathrm{~m}$ \\
Slot pitch & $\delta$ & $0.04 \mathrm{~m}$ & $0.04 \mathrm{~m}$ \\
\hline
\end{tabular}




\begin{tabular}{|c|c|c|c|}
\hline $\begin{array}{l}\text { Half-length from the } \\
\text { center of the duct to } \\
\text { the iron surface }\end{array}$ & $a$ & $0.21 \mathrm{~m}$ & $0.21 \mathrm{~m}$ \\
\hline $\begin{array}{r}\text { magnetic flux } \\
\text { density }\end{array}$ & $B$ & & \\
\hline $\begin{array}{r}\text { Linear current } \\
\text { density }\end{array}$ & $J$ & & \\
\hline $\begin{array}{l}\text { Half the duct } \\
\text { Thickness }\end{array}$ & $d$ & $0.2 \mathrm{~m}$ & $0.2 \mathrm{~m}$ \\
\hline width of water duct & $W$ & $1 \mathrm{~m}$ & $1 \mathrm{~m}$ \\
\hline $\begin{array}{l}\text { width of primary iron } \\
\text { of the machine }\end{array}$ & $w_{i}$ & $1 \mathrm{~m}$ & $1 \mathrm{~m}$ \\
\hline $\begin{array}{r}\text { Magneto motive } \\
\text { force }\end{array}$ & $M M F$ & & \\
\hline $\begin{array}{r}\text { Number of } \\
\text { conductors per coil } \\
\text { side }\end{array}$ & $n_{c}$ & 4 & 4 \\
\hline $\begin{array}{r}\text { Number of conductor } \\
\text { per slot }\end{array}$ & $n_{s}$ & 4 & 4 \\
\hline sea water speed & $V$ & $\begin{array}{l}0,0.02 \mathrm{Vs} \\
\ldots, 1.4 \mathrm{Vs}\end{array}$ & $\begin{array}{l}0,0.02 \mathrm{Vs}, \\
\ldots, 1.4 \mathrm{Vs}\end{array}$ \\
\hline $\begin{array}{l}\text { Synchronous speed } \\
\text { of the machine }\end{array}$ & $V_{s}$ & $3072 \mathrm{~m} / \mathrm{s}$ & $1536 \mathrm{~m} / \mathrm{s}$ \\
\hline wave number & $v$ & $\begin{array}{c}-7.55,- \\
7.52, \ldots \\
7.55\end{array}$ & $\begin{array}{c}-7.55,- \\
7.52, \ldots \\
7.55\end{array}$ \\
\hline $\begin{array}{r}\text { Average Electrical } \\
\text { Conductivity of sea } \\
\text { water }\end{array}$ & $\sigma$ & 10 & 10 \\
\hline effective current & $I$ & $1000 \mathrm{~A}$ & $1000 \mathrm{~A}$ \\
\hline Machine length & $\mathrm{L}$ & $122.9 \mathrm{~m}$ & $7.68 \mathrm{~m}$ \\
\hline
\end{tabular}




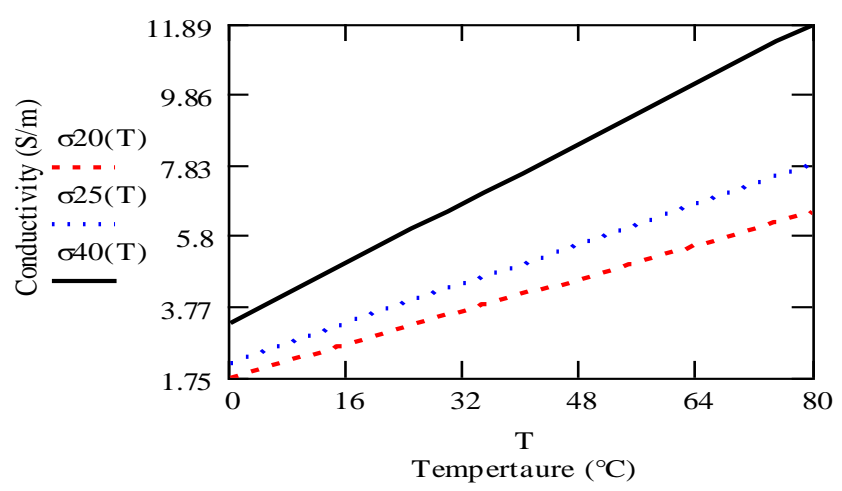

Fig. (2): Seawater conductivity versus temperature for different salinities 20,25 , and $40 \mathrm{~g} \mathrm{~kg}-1$

\section{Theory}

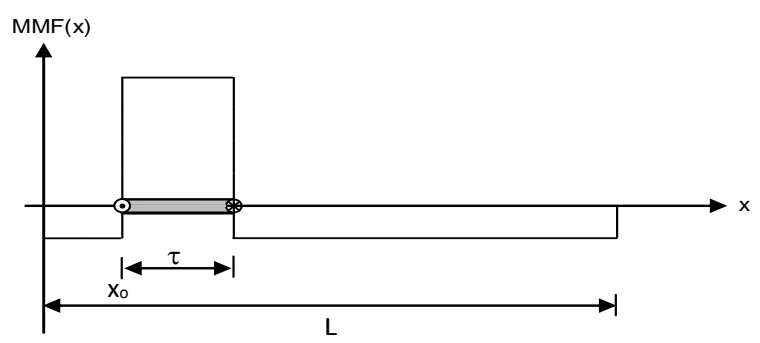

Fig. (3): MMF distribution of a single coil

Figure 3 shows the mathematical model for the discontinuous magneto motive force function of a single coil $\left(M M F_{c}\right)$ of slot pitch $\tau$ and length $L$ and primary current (i.e. effective current $I$ ). Mathematically, it is represented as;
The discontinuous function of the $\operatorname{MMF}(x, t)$ distribution for all $n_{c}$ coils of the 3-phase single layer winding with $2 p$-pole and with $q$-coils per phase per side is represented by Fourier integral as;

$$
M M F(x, t)=\frac{1}{2 \tau} \int_{-\infty}^{\infty} \overline{M M F}(v) e^{j\left(\frac{v \pi}{\tau} x-\omega t\right)} d v
$$

Where the Fourier transform $(\overline{M M F}(v))$ of the $M M F(x, t)$ in terms of the wavenumber, $v$, is given by;

(2)

$$
\overline{M M F}(v)=j \frac{n_{c} I \tau p q e^{j \omega t}}{\pi}\left(k_{P} k_{q} \frac{\left(e^{-j v \pi}-1\right)\left(1-e^{-j \pi(v-1)}\right)}{v\left(1-e^{-j \frac{\pi}{3}(v-1)}\right)}+\frac{\left(1-e^{-j \frac{v \pi}{\tau} L}\right)}{v\left(1-e^{-j \frac{\pi}{3}}\right) p}\right)
$$

Where; 
$k_{q}(v)=\frac{1-e^{-j \frac{v \pi q \delta}{\tau}}}{q\left(1-e^{-j \frac{v \pi \delta}{\tau}}\right)}$

$k_{p}(v)=\frac{1-e^{-j 2 \pi v p}}{p\left(1-e^{-j 2 \pi v}\right)}$

and $\omega=2 \pi H z$, and $\delta=\tau / 3 q$ is the slot pitch (for a 3-phase system). Figure (4) shows the stepped $\operatorname{MMF}(x, t)$ wave for machine with $q=4$ slots at two instants 0 and $1 / 4$ the period with frequency $800 \mathrm{~Hz}$.

The relation between the linear current density, $J_{z}$, and the $M M F$ is derived from Ampere's circuital law, i.e.

$J_{z}(x)=\frac{\partial}{\partial x} \operatorname{MMF}(x)$

Applying Fourier transform (FT) to (5) with respect to the $x$ - coordinate, we get

$$
\bar{J}_{z}(v)=j \frac{v \pi}{\tau} \overline{M M F}(v)
$$

Substituting (4) into (6) which can be approximated to

$$
\bar{J}_{z}(v)=j \frac{J \tau}{\pi(v-1)}\left(e^{-j 2 \pi v p}-1\right)
$$

Where

$$
J=j \frac{3 q n_{c} I k_{q}}{\tau} e^{-j\left(\frac{\pi}{2 \tau}(\tau+(q-1) \delta-\omega t)\right.}
$$

\section{Assumptions}

In the following work we will consider the following assumptions:

- The seawater temperature is constant.

- The primary iron core has very high relative permeability $\mu_{r}$ compared to vacuum permeability $\mu_{0}$.

- A laminar flow of seawater enters the water duct.

- The overhang of the machine winding is incorporated into the model.

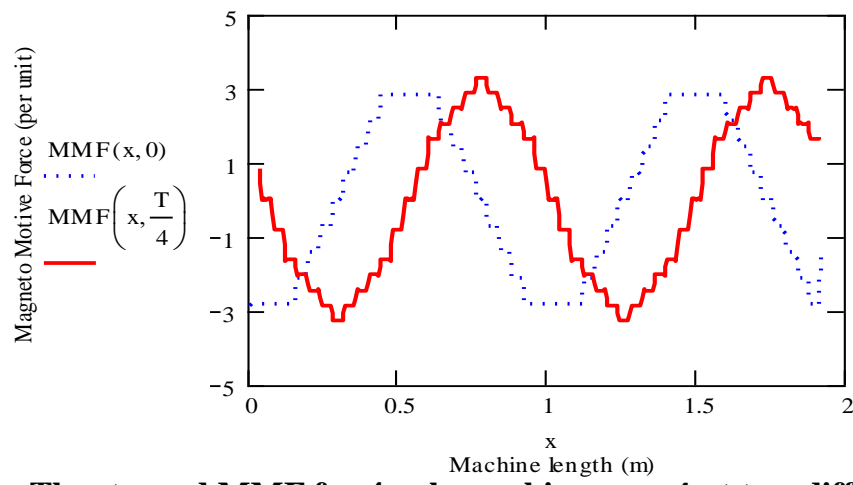

Fig. (4): The stepped MMF for 4 pole machine, $q=4$ at two different instants, $t=0, \frac{T}{4} \mathrm{~s}$.

\section{Field Analysis}

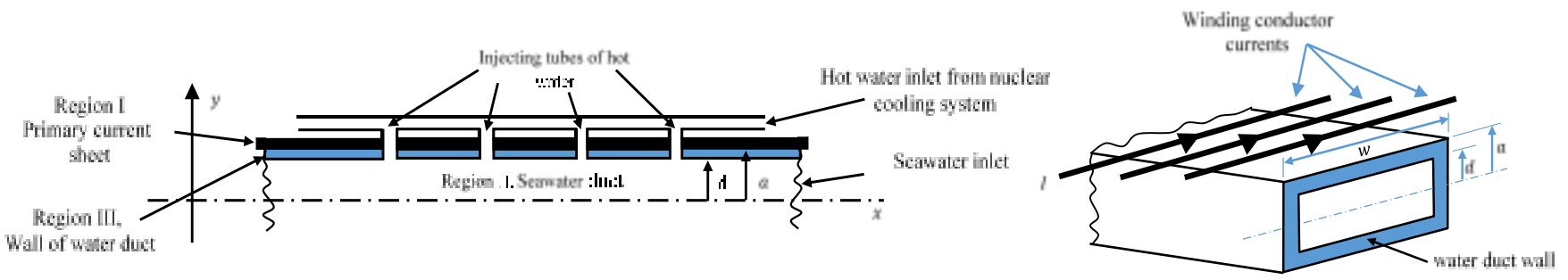

(a)

(b)

Fig. (5): Mathematical model of the LID.

(a) Sectional view. (b) A simplified 3D - sketch for the duct and conductors 
Figure $5 \mathrm{a}$ is a sectional view of the mathematical model of the LID showing the insertion of hot water tubes from the nuclear coolant system into the seawater duct of the LID to insure the uniformity of hot water distribution to improve seawaters conductivity. The water duct tempertaure can be controlled by increasing the number of injecting tubes. Figure $5 b$ shows a simplifed 3D-view of the water duct and the winding conductor currents. Based on Maxwell's field equations, and ohm's law, the basic partial differential equation of the magnetic vector potential $A$ in each region will take the form, see Figure 5a,

$$
\nabla^{2} A=\mu_{o} \sigma \frac{\partial A}{\partial t}
$$

Using the appropriate boundary conditions, solving (8) for the $z$-component of the magnetic vector potential, $A \equiv A\left(0,0, A_{z}\right)$, in region II (i.e. seawater) in transformed form we get,

$$
\bar{A}_{2}=\mu_{o} \frac{\bar{J}_{z}}{H(v)} G(v, y)
$$

Where

$$
G(v, y)=\cosh \gamma d \cosh \left(\frac{v \pi}{\tau}\right)(y-d)-\frac{\mu_{o} \tau \gamma}{\mu_{2} v \pi} \sinh \gamma d \sinh \left(\frac{v \pi}{\tau}\right)(y-d)
$$

And

And

$$
\gamma=\sqrt{\left(\frac{v \pi}{\tau}\right)^{2}+j \frac{\mu \sigma \pi}{\tau}(v V-2 \tau H z)}
$$

$$
H(v)=\frac{v \pi}{\tau} \cosh \gamma d \sinh \left(\frac{v \pi}{\tau}\right)(a-d)+\frac{\mu_{o}}{\mu_{2}} \gamma \sinh \gamma d \cosh \left(\frac{v \pi}{\tau}\right)(a-d)
$$

Where $\mu_{2}$ is the magnetic permeability of the sea water, imperically it equals $\mu_{0}$. The magnetic induction, $B \equiv B\left(B_{x}, B_{y}, 0\right)$, can be derived usig the relation $B=\nabla \times A$ and the transformed form of the $\mathrm{y}$-component in region II will be given by;

$$
\bar{B}_{2 y}(v)=-j \mu_{o} \frac{v \pi G(v, y)}{\tau H(v)} \bar{J}_{z}(v)
$$

The electric field strength has only a $z-$ component, $E \equiv E\left(0,0, E_{z}\right)$, and its relation to the magnetic vector potential in region II is given by

$$
E_{2 z}=-\frac{\partial A_{2}}{\partial t}
$$

In terms of the FT it will be

$$
\bar{E}_{2 z}=-j \omega \bar{A}_{2}
$$

\section{The force expression}

In the model shown in Figure 5b, the primary current, $I$, flows in the negative $z$-direction. Then, the thrust force in the $x$-direction will be given by

$$
F_{x}=\frac{W}{4 \tau} \operatorname{Re} \int_{-\infty}^{+\infty} \bar{B}_{y}(v) \bar{J}_{z}^{*}(v) d v
$$

Where, "Re" means the real part of the thrust force and $W$ is the width of the water duct and $\bar{J}_{z}^{*}(v)$ is the transformed conjugate linear current density, and $\bar{B}_{y}(v)$ is the y-component of the transformed magnetic induction, which are given by

$$
\bar{J}_{z}^{*}(v)=-j \frac{J \tau}{\pi(v-1)}\left(e^{j 2 \pi v p}-1\right)
$$

and

$$
\bar{B}_{2 y}(v)=-j \mu_{o} \frac{v \pi G(v, y)}{\tau H(v)} \bar{J}_{z}(v)
$$

Analytic force expression

Substituting (16) and (17) into (15), and after tedious derivation, we get the analytical expression for the force,

$F_{x}=\frac{\mu_{o} w}{2}\left[\frac{3 q n_{c} I k_{q}}{\tau}\right]^{2} \operatorname{Re}[D-2 \pi j(F-E)]$

Where $D, E$, and $F$ are given by,

$$
D=\frac{2 \pi p G(1, y)}{j H(1)} \quad E=\frac{v_{o}\left(1-e^{2 \pi j v_{o} p}\right) G\left(v_{o}, y\right)}{\left(v_{o}-1\right)^{2} H^{\prime}\left(v_{o}\right)} \quad F=\frac{v_{o}^{\prime}\left(1-e^{-j 2 \pi v_{o}^{\prime} p}\right) G\left(v_{o}^{\prime}, y\right)}{\left(v_{o}^{\prime}-1\right)^{2} H^{\prime}\left(v_{o}^{\prime}\right)}
$$


and

$$
v_{o}, v_{o}^{\prime}=\frac{j \mu_{o} \sigma \pi V d}{2 \tau\left(\frac{\pi^{2}}{\tau^{2}}(a-d)+\frac{\mu_{o} d \pi^{2}}{\mu_{2} \tau^{2}}\right)} \pm \sqrt{-\frac{\mu_{o}^{2} \sigma^{2} \pi^{2} V^{2} d^{2}}{4 \tau^{2}\left(\frac{\pi^{2}}{\tau^{2}}(a-d)+\frac{\mu_{o} d \pi^{2}}{\mu_{2} \tau^{2}}\right)^{2}}-\frac{j \mu_{o} \sigma \pi V_{s} d}{\tau\left(\frac{\pi^{2}}{\tau^{2}}(a-d)+\frac{\mu_{o} d \pi^{2}}{\mu_{2} \tau^{2}}\right)}}
$$

and

$$
V_{s}=2 \tau H z
$$

is the synchronous speed.

\section{Numerical Force Expression}

The numerical force expression has been calculated by the program MATHCAD and the limits have been modified from $(-\infty$ to $+\infty)$ to $(-8.5$ to 8.5$)$ which gives enough accuracy in this range as the harmonics beyond this period are very small and can be neglected. In the following calculations we used the parameters given in table I. Equation (15) can be expressed as

$$
F_{x}=\int_{-\infty}^{+\infty} C M(v, V) S(v) d v
$$

Where

$$
S(v)=\frac{\tan (2 \pi p v)-\sin (2 \pi p v)}{\pi(v-1)^{2} \tan (2 \pi p v)}
$$

and

$$
C M(v, V)=\left[\frac{\mu w}{2}\left(\frac{m n_{s} q I_{p} k_{q}}{\tau}\right)^{2}\right] \operatorname{Re}\left(\frac{v G(v, V)}{j H(v, V)}\right)
$$

Where $H(v, V)$ is given by (11) as $\gamma$ includes the speed $V$.

\section{Calculation of seawater input power}

The seawater input power $\left(P_{S W}\right)$ will take the form

$$
P_{\mathrm{s} w}=\frac{W}{2(2 \tau)} \operatorname{Re} \int_{-\infty}^{+\infty} \bar{J}_{z}^{*}(v) \bar{E}_{z}(v) d v
$$

By substituting (14) and (16) into (24) we get the analytical expression of the seawater input power

$$
P_{\mathrm{sw}}=\frac{W V_{s} \mu_{o}}{2}\left[\frac{3 q n_{c} I_{m} k_{\omega 1}}{\tau}\right]^{2} \operatorname{Re}(A+B-C)
$$

Where $A, B$, and $C$ are given by

$$
A=\frac{2 \pi p}{j} \frac{G(1, y)}{H(1)}
$$

$$
\begin{gathered}
B=\frac{\left(1-e^{2 \pi j v_{o} p}\right) G\left(v_{o}, y\right)}{\left(v_{o}-1\right)^{2} H^{\prime}\left(v_{o}\right)} \\
C=\frac{\left(1-e^{-2 \pi j v_{o}^{\prime} p}\right) G\left(v_{o}^{\prime}, y\right)}{\left(v_{o}^{\prime}-1\right)^{2} H^{\prime}\left(v_{o}^{\prime}\right)}
\end{gathered}
$$

Where $H^{\prime}\left(v_{o}^{\prime}\right)$ is the derivative of $H\left(v_{o}^{\prime}\right)$ with respect to $v$.

\section{Efficiency and the Power Factor}

The efficiency of the LID is given by $\eta=\frac{\text { Seawater Output power }}{\text { Seawater Input power }}$

$\eta=\frac{V}{V_{s}} \frac{\operatorname{Re}\left\{\frac{2 \pi p G(1, y)}{j H(1)}+\frac{v_{o}\left(1-e^{2 \pi j v_{o} p}\right) G\left(v_{o}, y\right)}{\left(v_{o}-1\right)^{2} H^{\prime}\left(v_{o}\right)}-\frac{v_{o}^{\prime}\left(1-e^{-2 \pi j v_{o}^{\prime} p}\right) G\left(v_{o}^{\prime}, y\right)}{\left(v_{o}^{\prime}-1\right)^{2} H^{\prime}\left(v_{o}^{\prime}\right)}\right\}}{\operatorname{Re}\left\{\frac{2 \pi p G(1, y)}{j H(1)}+\frac{\left(1-e^{2 \pi j v_{o} p}\right) G\left(v_{o}, y\right)}{\left(v_{o}-1\right)^{2} H^{\prime}\left(v_{o}\right)}-\frac{\left(1-e^{-2 \pi j v_{o}^{\prime} p}\right) G\left(v_{o}^{\prime}, y\right)}{\left(v_{o}^{\prime}-1\right)^{2} H^{\prime}\left(v_{o}^{\prime}\right)}\right\}}$

Whereas the power factor $(P F)$ is given by;

$$
P F=\frac{\text { Seawater Input power }}{\mid \text { Seawater Input power } \mid}
$$

The improved power factor ( IPF ) can be obtained by adding a capacitor bank in series to the primary current sheet to eliminate the reactance of the winding of LID, which reduces the applied voltage, then complex seawater input power $\left(I P_{s w}\right)$

$$
I P_{s w}=\operatorname{complex}\left(P_{s w}\right)-j \operatorname{Im}\left(\operatorname{complex}\left(P_{s w}\right)\right)
$$

And the IPF will be given by

$I P F=\frac{\text { improved seawater input power }}{\mid \text { improved seawater input power } \mid}$ 


\section{Results and Discussion}

In this section we will analyze our results in case of both long and short machines.

\section{Long machine}

Thrust force:

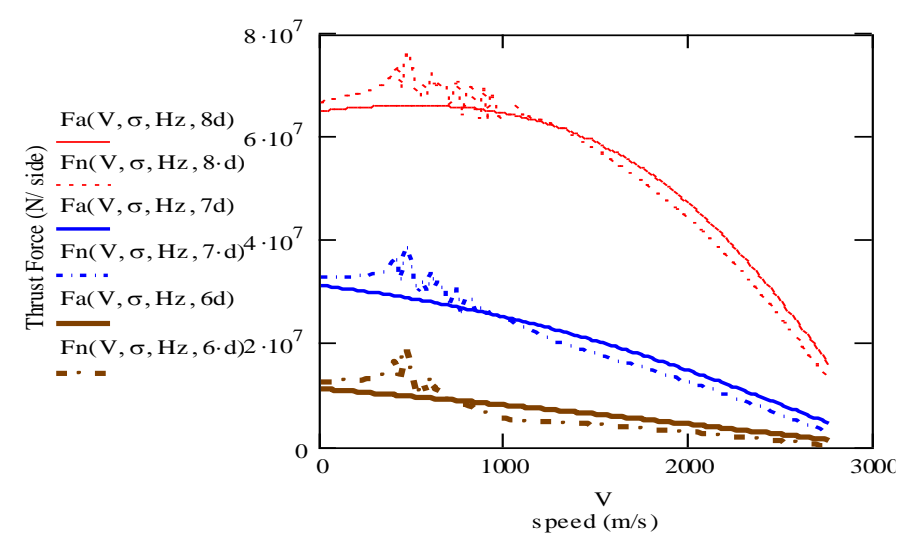

Fig. (6a) A comparison between the analytical (Fa) and numerical (Fn) thrust force - speed curve, for 3 duct sizes $6 d, 7 d$ and $8 d$, (where $d=0.2 \mathrm{~m}$ )

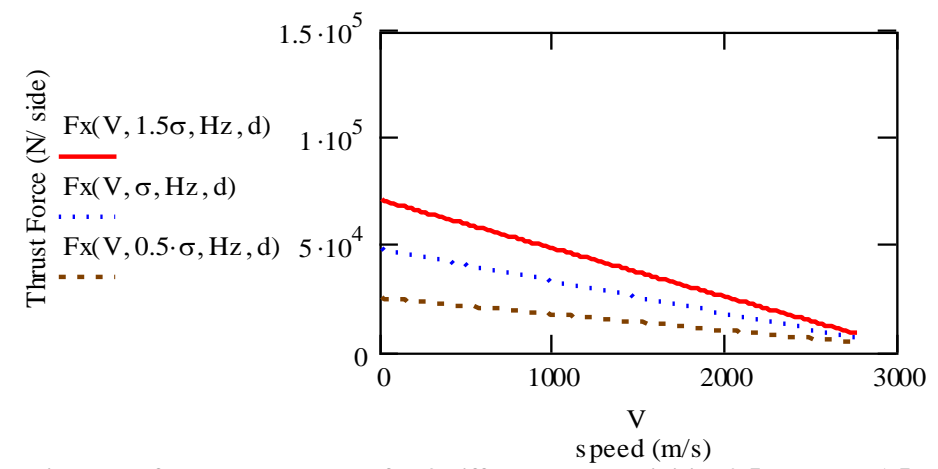

Fig (6b). The analytic thrust force - speed curve for 3 different conductivities $0.5 \sigma, \sigma$, and $1.5 \sigma$ (where $\sigma=10 \Omega^{-1} \mathrm{~m}^{-1}$ )

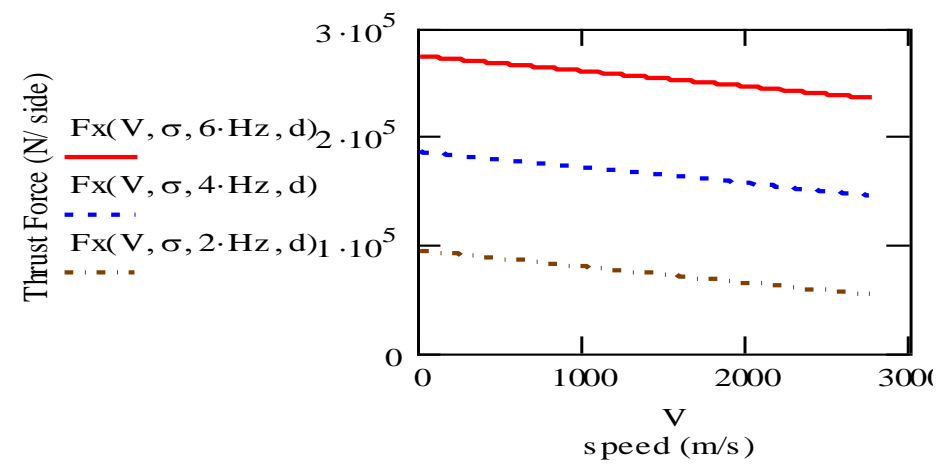

Fig. (6c): The analytic thrust force - speed curve for 3 different frequency $2 \mathrm{~Hz}, 4 \mathrm{~Hz}$, and $6 \mathrm{~Hz}$, (where $\mathrm{Hz}=800 \mathrm{~s}^{-1}$ ) 


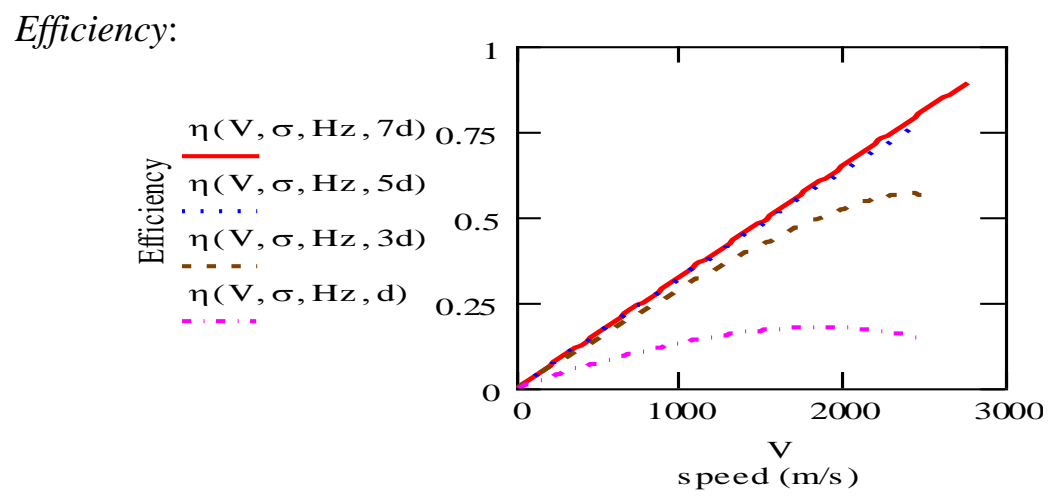

Fig. (7a): The effect of duct size on the efficiency - speed curve for 4 different duct sizes $d, 3 d, 5 d$, and $7 d$ (where $\mathbf{d}=0.2 \mathrm{~m})$

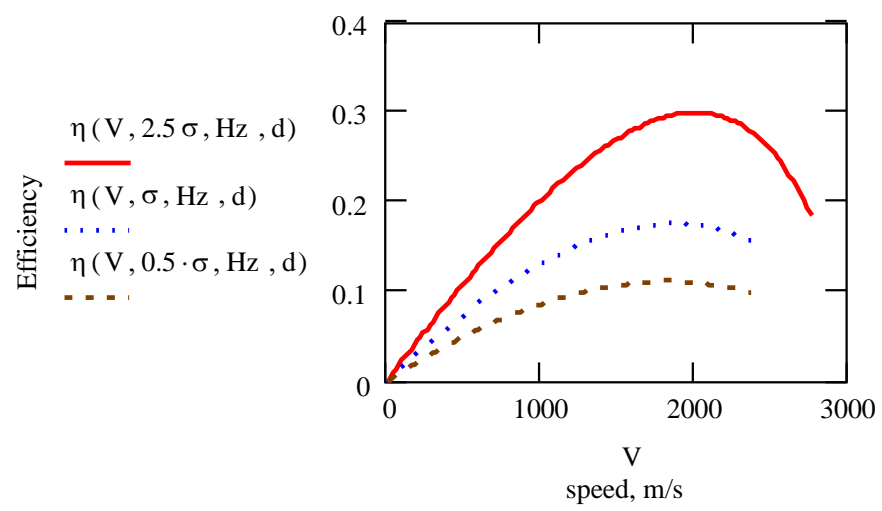

Fig. (7b): The effect of conductivity on the efficiency-speed curve for 3 different conductivities $0.5 \sigma, \sigma$, and $2.5 \sigma$, (where $\sigma=10 \Omega^{-1} \mathbf{m}^{-1}$ )

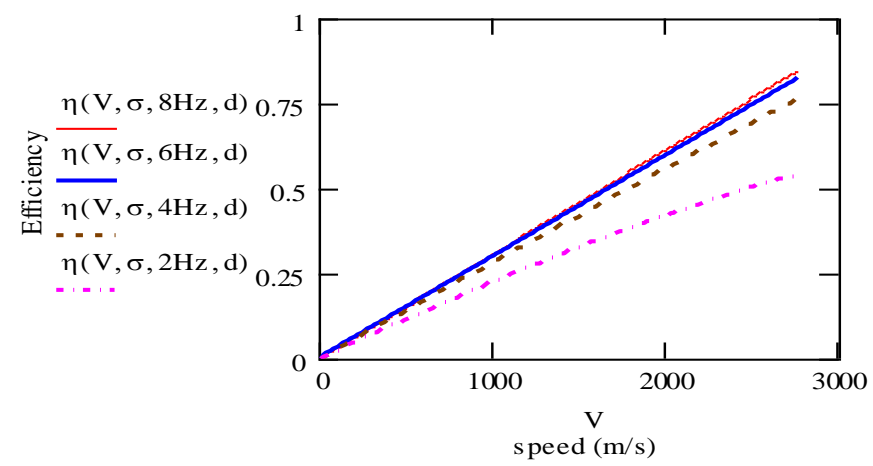

Fig. (7c): The effect of varying the frequency (i.e. $2 \mathrm{~Hz}, 4 \mathrm{~Hz}, 6 \mathrm{~Hz}$, and $8 \mathrm{~Hz}$ ) on the efficiency-speed curve. 
Power factor:

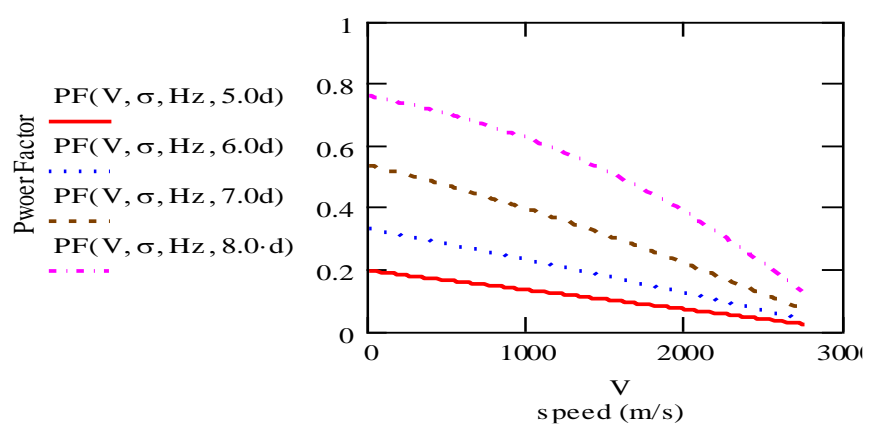

Fig. (8a): The effect of varying the duct size on the power factor for 4 different duct sizes $5 d, 6 d, 7 d$, and $8 d$ $($ where $\mathrm{d}=0.2 \mathrm{~m})$

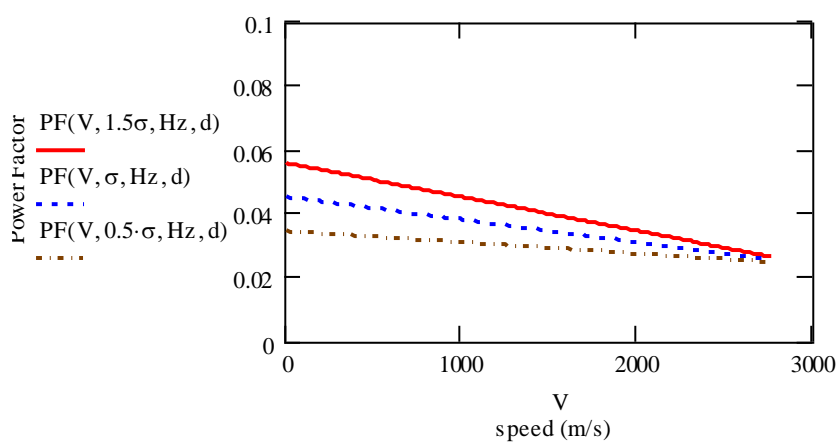

Fig. (8b): The effect of conductivity on the power factor using 3 different conductivities $0.5 \sigma, \sigma$, and $1.5 \sigma$, (where $\sigma=10 \Omega^{-1} \mathbf{m}^{-1}$ )

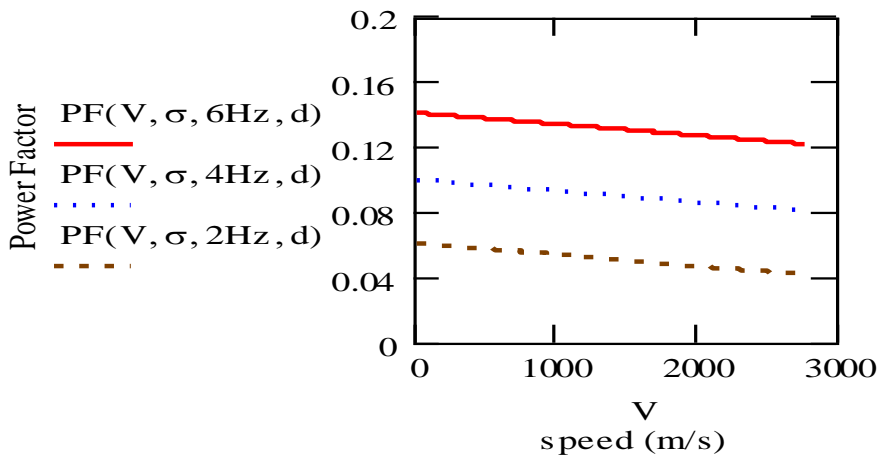

Fig. (8c): Shows the effect of varying the frequency on the power factor for 3 different frequencies $2 \mathrm{~Hz}, 4 \mathrm{~Hz}$, and $6 \mathrm{~Hz}$, (where $\mathrm{Hz}=800 \mathrm{~s}^{-1}$ ) 
Short Machine

a) Thrust force:

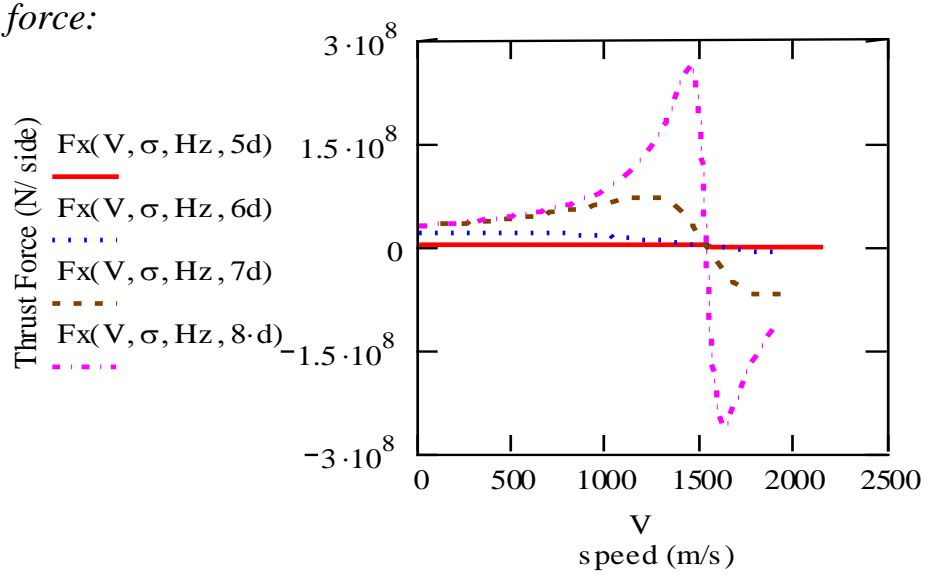

Fig. (9a): The effect of varying the duct size on the thrust force for 4 different duct sizes $5 d, 6 d, 7 d$, and $8 d$ $($ whered $=0.2 \mathrm{~m})$

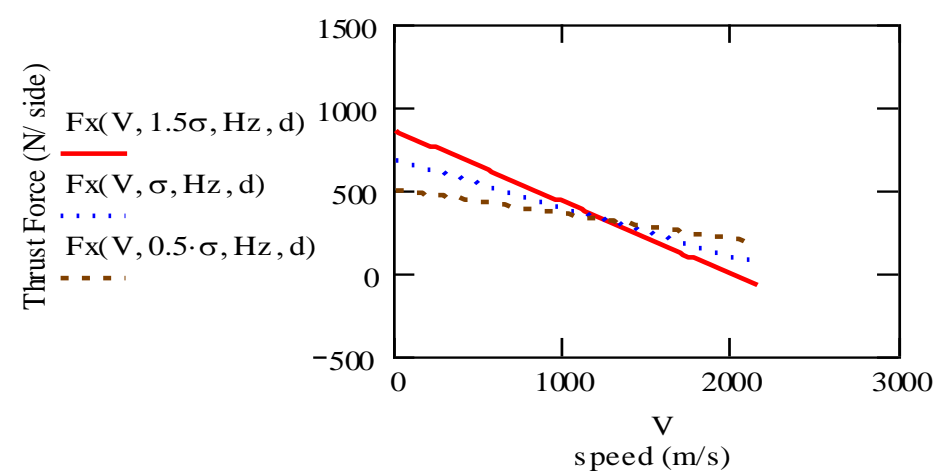

Fig. (9b): The effect of conductivity on the thrust force for 3 different conductivities $0.5 \sigma, \sigma$, and $1.5 \sigma$, (where $\sigma=10 \Omega^{-1} \mathbf{m}^{-1}$ )

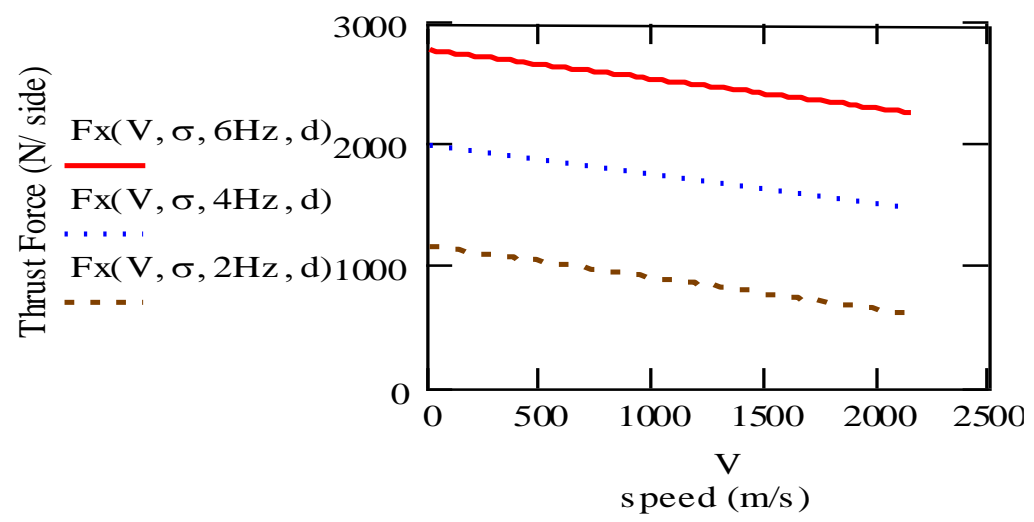

Fig. (9c): The effect of frequency on the thrust force for $3 \mathrm{different}$ frequencies $2 \mathrm{~Hz}, 4 \mathrm{~Hz}$, and $6 \mathrm{~Hz}$, (where $\mathrm{Hz}=800 \mathrm{~s}^{-1}$ ) 
b) Efficiency:

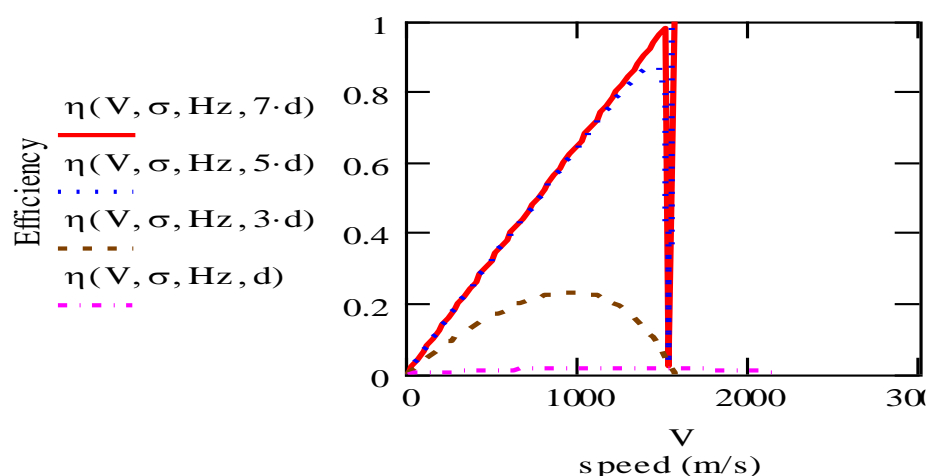

Fig. (10a): The effect of duct size on the efficiency - speed curve for 4 different duct sizes $d, 3 d, 5 d$, and $7 d$ (where $\mathbf{d}=\mathbf{0 . 2} \mathbf{m})$

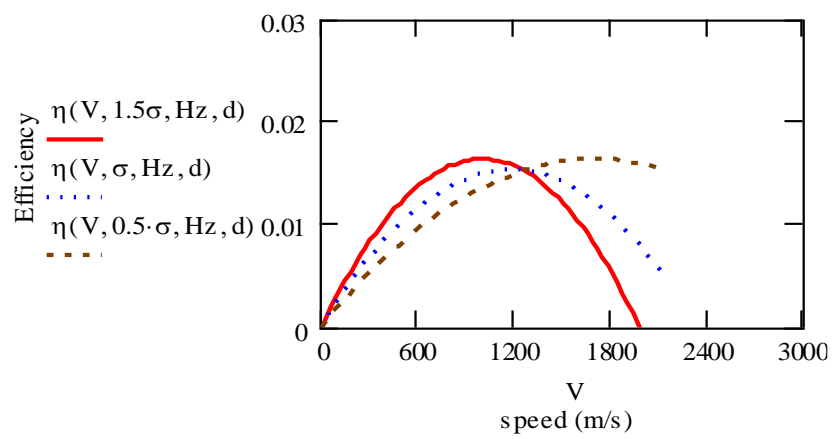

Fig. (10b): The effect of conductivity on the efficiency - speed curve for 3 different conductivities $0.5 \sigma, \sigma$, and $1.5 \sigma,\left(\right.$ where $\left.\sigma=10 \Omega^{-1} \mathrm{~m}^{-1}\right)$

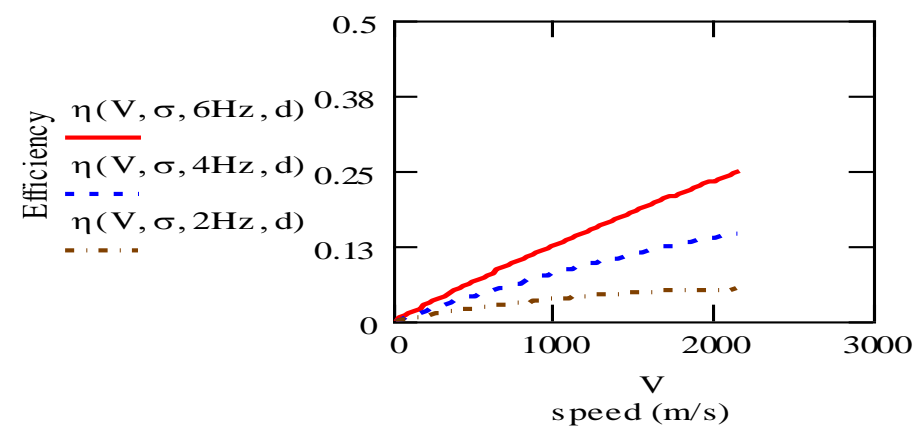

Fig. (10c): The effect of varying the frequency (i.e. $2 \mathrm{~Hz}, 4 \mathrm{~Hz}$, and $6 \mathrm{~Hz}$ ) on the efficiency - speed curve 
c) Power factor:

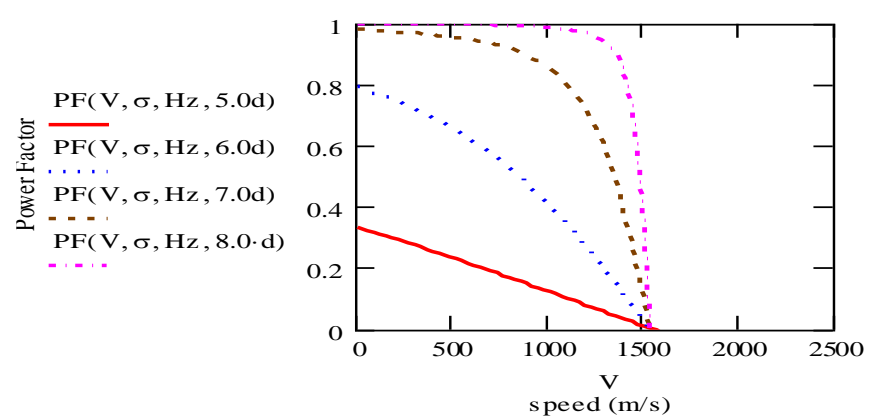

Fig (11a): The effect of varying the duct size on the power factor - speed curve for 4 different duct sizes $5 d, 6 d, 7 d$, and $8 d($ where $d=0.2 \mathrm{~m})$

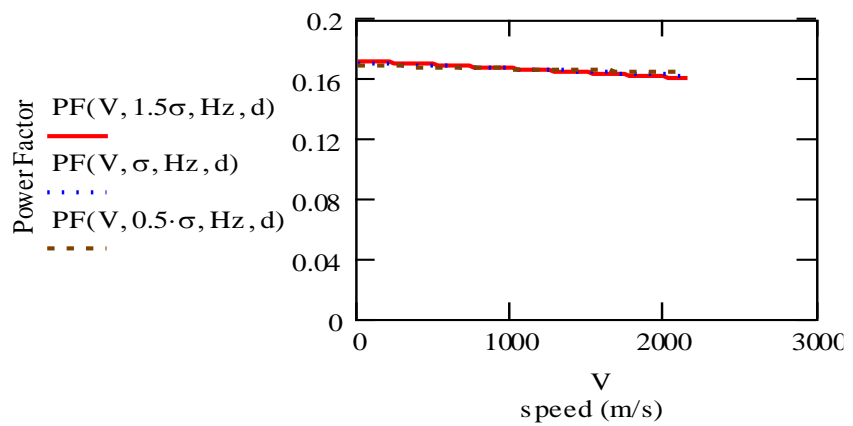

Fig (11b): The effect of conductivity on the power factor - speed curve for 3 different conductivities $0.5 \sigma, \sigma$, and $1.5 \sigma,\left(\right.$ where $\left.\sigma=10 \Omega^{-1} \mathrm{~m}^{-1}\right)$

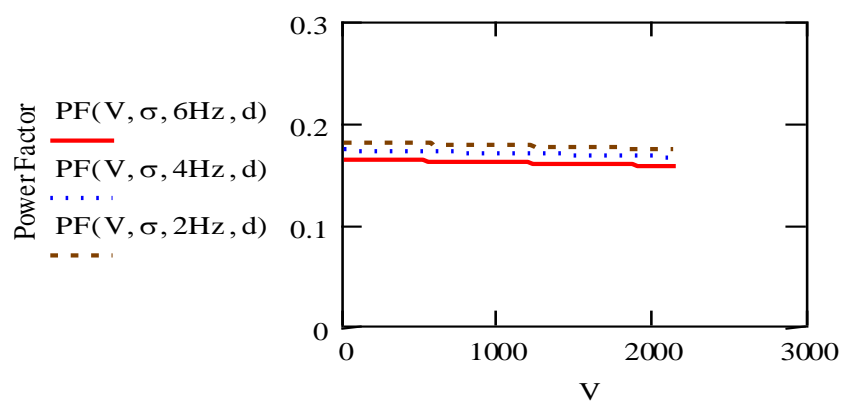

Fig (11c): Shows the effect of varying the frequency on the p 8 wet factor - speed curve for 3 different frequencies $2 \mathrm{~Hz}, 4 \mathrm{~Hz}$, and $6 \mathrm{~Hz}$, (where $\mathrm{Hz}=800 \mathrm{~s}^{-1}$ )

In case of the long machine, Figure (6a) shows a comparison between the analytical $(\mathrm{Fa})$ and numerical (Fn) thrust force - speed curve along the $\mathrm{x}$-axis, for 3 different duct sizes $6 d, 7 d$ and $8 d$, (where $d=0.2 \mathrm{~m}$ ). The Figure indicates that as the duct size increases linearly (i.e. $6 d, 7 d$ and $8 d$ ) the thrust force - speed curve increases nonlinear (approximately $1.2 \times 10^{7}, 3.1 \times 10^{7}, 6.8 \times 10^{7} \mathrm{~N}$ ) respectively. The ripples appearing in the numerical solutions are due to the existence of the higher harmonic orders of the MMF. Figure 9a depicts the analytic thrust force - speed curve for 4 different duct sizes $5 d, 6 d, 7 d$ and $8 d$, (where $d=0.2 \mathrm{~m}$ ) in case of short machine. The figure shows as the duct size increase linearly $(5 d, 6 d, 7 d$, and $8 d)$, the initial thrust force increases rapidly to become approximately constant (i.e. $3.26 \times 10^{6}, 1.85 \times$ $10^{7}, 2.85 \times 10^{7}, 2.95 \times 10^{7}$ ) at about $7 d$ to $8 d$ respectively, and that is due to the decay of the field in the duct. So when considering the design, $7 d$ should be considered. Figures 6b-c (long machine) and 9b-c (short machine) show the analytic thrust force - speed curve for 3 different conductivities $0.5 \sigma, \sigma$, and $1.5 \sigma$ (where $\sigma=10 \Omega^{-1} m^{-1}$ ) and 3 different frequencies $2 \mathrm{~Hz}, 4 \mathrm{~Hz}$, and $6 \mathrm{~Hz}$, (where $\mathrm{Hz}=800 \mathrm{~s}^{-1}$ ) respectively. In case of long (figure 
6b) and short (figure 9b) machine it is clear that, as the conductivity increases linearly $(0.5 \sigma, \sigma$, and $1.5 \sigma)$ the thrust force - speed curve increases linearly with increasing negative slopes and approximately constant synchronous speed $V_{s}$. This shows that the variation of the conductivity increases the initial thrust force, while preserving the synchronous speed. While in Figs.6c (long machine) and 9c (short machine) as the frequency increases linearly $(2 \mathrm{~Hz}, 4 \mathrm{~Hz}$, and $6 \mathrm{~Hz})$ the thrust force - speed curve increases linearly with constant slopes and increasing synchronous speed $V_{s}$. Figures $7 \mathrm{a}-\mathrm{c}$ and $10 \mathrm{a}-\mathrm{c}$ show the effect of duct size, conductivity, and frequency on the efficiency speed curve respectively. From Figs. 7a (long machine) and 10a (short machine), it is clear that the variation of duct size $(d, 3 d, 5 d$, and $7 d$ where $d=$ $0.2 \mathrm{~m}$ ) has a significant effect on the efficiency speed curve and becomes constant at approximately $5 \mathrm{~d}$ due to the decay of the field in the duct. While figure $7 \mathrm{~b}$ (long machine) shows a significant increase on the efficiency - speed curve due to the variation of conductivity $(0.5 \sigma, \sigma$, and $2.5 \sigma$, where $\sigma=10 \Omega^{-1} \mathrm{~m}^{-1}$ ) on the contrary to the case of short machine, figure $10 \mathrm{~b}$, which shows a slit increases on the efficiency - speed curve due to the variation of conductivity. From this, it is clear that the variation of conductivity is more effective on the long machines than on the short machines.

Figure 7c (long machine) depicts the effect of varying the frequency $\left(2 \mathrm{~Hz}, 4 \mathrm{~Hz}, 6 \mathrm{~Hz}\right.$, and $8 \mathrm{~Hz}$ where $\mathrm{Hz}=800 \mathrm{~s}^{-1}$ ) on the efficiency - speed curve, which shows that as the frequency increases the efficiency - speed curve increases till becoming approximately constant at about $6 \mathrm{~Hz}$, with a high peak efficiency $(>80 \%)$. on the contrary to the case of short machines, figure 10c, which shows an increase in efficiency - speed curve with the increase of frequency $(2 \mathrm{~Hz}, 4 \mathrm{~Hz}, 6 \mathrm{~Hz})$ but with low peak efficiency $(\sim 30 \%)$.

Figures $8 \mathrm{a}-\mathrm{c}$ and $11 \mathrm{a}-\mathrm{c}$ show the effect of varying the duct size, conductivity, and frequency on the power factor - speed curve respectively. It is clear from Figs. 8a (long machine) and 11a (short machine) that the power factor - speed curve is affected significantly by the variation of duct size, i.e. increasing duct size causes an increase in power factor- speed curve, while in case of short machine the variation of conductivity, figure $11 \mathrm{~b}$, and frequency, figure $11 \mathrm{c}$, the power factor - speed curve has no significant affect when varying both the conductivity and frequency. In case of long machine, when varying the conductivity, figure $8 \mathrm{~b}$, and frequency, figure 8c, both show an increase in the power factor - speed curve when increasing the conductivity and frequency respectively.

\section{Conclusion}

In this paper, a 2-dimensional theory (analytical and numerical) for linear induction drives (LIDs) using hot seawater from coolant system of nuclear reactor as a driving medium has been presented, this approach is feasible as we can get a considerable thrust force from these types of machines with proper efficiency, making this a new approach for silent propulsion systems. A wide range of analysis, helps choosing the optimal designs parameters required whether it is a nuclear submarine or a supercarrier. In addition, the study showed that for the same length of machine L, by decreasing the number of poles and increasing the pole pitch (long machine) this improves the performance rather than increasing the number of poles and decreasing the pole pitch (short machine). Compromising between the available technology and theory is essential depending on the budget and the design consideration. For future studies, the given approach in this paper paves the way to a new field of space rocket propulsion systems using plasma as a deriving medium.

\section{References}

1. Yoshi ANDO, (1969) Safety evaluation of the First Nuclear Ship in Japan, Nuclear Engineering and Design, 10(2), 243-249.

2. John Pike, (2006) US Navy Propulsion Systems - Federation of American Scientists. 1999-0227. Archived from the original on 25 September 2006. Retrieved 2006-10-16. Current cores for the NIMITZ Class aircraft carrier.

3. "A4W". US Navy Propulsion Systems. Federation of American Scientists. 1999-02-27. Archived from the original on 25 September 2006. Retrieved 2006-10-16.

4. Marian Piwowarski, (2013) The analysis of turbine propulsion systems in nuclear submarines, Key Engineering Materials, Vol. 597, 99-105.

5. Pai, R.M.; Boldea, I.; Nasar, S.A., (1988) A complete equivalent circuit of a linear induction motor with sheet secondary, IEEE Transactions on Magnetics, 24(1), $639-654$. 
6. Laithwaite E.R., (1955) Adapting a linear induction motor for the acceleration of large masses to high velocities Electric Power Applications, IEE Proceedings, 142(4), 262 268.

7. Mirsalim, M.; Doroudi, A.; Moghani, J.S., (2002) Obtaining the operating characteristics of linear induction motors: a new approach, IEEE Transactions on Magnetics, 38(2), 1365 -1370 .

8. Jamali J., (2003) End effect in linear induction and rotating electrical machines,IEEE

Transactions on Energy Conversion, 18(3), 440 - 447. DOI: 10.1109/TEC.2003.815853

9. Ramkumar, S. Balaji, M. Sivakumar, N. Kamaraj, V. (2006) Performance Evaluation of Single Sided Linear Induction Machine using Finite element Analysis. IEEE Xplore Digital Library, pp. 1080-1082. DOI: 10.1109/EPEPEMC.2006.4778545

10. Francesco Alonge, Maurizio Cirrincione, Filippo D'Ippolito, Marcello Pucci, Antonino Sferlazza, (2016) Adaptive feedback linearizing control of linear induction motor considering the end-effects, Control Engineering Practice, 5, 116-126.

11. M. E. Ismaeel, Supersonic Speed Ground Transportation and the Space Launcher, Poster Session Paper, Int. Conf. on Electrical Machines (ICEM), Lausanne-Switzerland, Sept. 18-21, 1984.

12. Sherif M. E. Ismaeel, Mostafa M. Shalaby, Mohammed F. Salem, Atef M. Khazbak, (2012), The 3-Dimensional Theory of Supersonic and Higher Speeds of Linear Induction Motors, International Journal of Engineering Studies, 4(3), 249 - 265.

13. Mostafa M. Shalaby, Mohammed F. Salem, Atef A. Khazbak, et al, (2010) Towards a Deep Understanding of the Stepped MMF Wave Packets of Linear Induction Machines, International Journal of Engineering Studies, 2(1), $89-103$.

14. M. E. Ismaeel, (1978) Theories of Linear Induction Motors With Completely-filled and Half-filled Primary End-Slots, Proc. IEE, 125(7), 657-665.

15. M. E. Ismaeel (1978) Traveling Waves in Linear Induction Machines, Proc. IEE, 125(6), 527-528.

16. Sir Edward Bullard, "Physical properties of sea water," National Physical Laboratory,
17. http://www.kayelaby.npl.co.uk/general_ph ysics/2 7/2 7 9.html

18. M. Hill (ed.), (1970), the Sea, vol. 1, chap. 1 and vol. 4, pt. 1, chap. 18 (Wiley) and Cox et al., Deep Sea Res., 17, 679. 